\title{
PERFIL DAS CRIANÇAS USUÁRIAS DO AMBULATÓRIO DE SAÚDE MENTAL DO MUNICÍPIO DE IÇARA - SC
}

\author{
Maísa Pedro da Silva Serafim \\ Universidade do Extremo Sul de Santa Catarina (UNESC) \\ Karin Martins Gomes \\ Universidade do Extremo Sul de Santa Catarina (UNESC) \\ Dipaula Minotto da Silva \\ Universidade do Extremo Sul de Santa Catarina (UNESC) \\ João Luiz Brunél \\ Universidade do Extremo Sul de Santa Catarina (UNESC)
}

\begin{abstract}
Resumo
O objetivo deste estudo foi delinear o perfil das crianças atendidas no Ambulatório de Saúde Mental de Içara - SC. Portanto, realizou-se uma pesquisa documental, de abordagem quantitativa e de natureza exploratória. A coleta de dados deu-se através dos prontuários das crianças de 6 a 11 anos atendidas no período de 2013 a junho de 2016. Observou-se que 58,3\% eram do sexo masculino, 38,3\% tem idade entre 8 e 9 anos e $27 \%$ são encaminhados por suspeita de transtorno do neurodesenvolvimento. Sobre o trabalho realizado no local, pode-se inferir que $53 \%$ são atendidas pela psicóloga, $31 \%$ fazem psicoterapia, $21 \%$ usam medicação e $12 \%$ associam psicoterapia com psicofármaco. É necessário pensar em ações para esta demanda sob uma lógica biopsicossocial. Conhecer o usuário torna possível encontrar subsídios para repensar as práticas atuais e formular melhores métodos de atuação.
\end{abstract}

Palavras chaves: políticas públicas; infância; saúde mental.

\section{PROFILE OF CHILDREN ATTENDED AT THE IÇARA MENTAL HEALTH CLINIC - SC}

\begin{abstract}
This study aimed to outline the profile of the children attending the Mental Health Outpatient Clinic of Içara SC. For that, a documentary research, of quantitative approach and of exploratory nature, was carried out. Data collection was done through the records of children aged 6 to 11 years old between 2013 and June 2016. It was observed that $58.3 \%$ were males, $38.3 \%$ are between 8 and 9 years of age and $27 \%$ are referred for suspected Neurodevelopmental disorder. In this local $53 \%$ are cared for by the psychologist, 31\% make psychotherapy, $21 \%$ use medication and $12 \%$ associate psychotherapy with psychotropic drugs. However, it is necessary to think about practices for this demand under biopsychosocial logic. Knowing the user becomes possible subsidy to rethink current practices and formulate better methods of action.
\end{abstract}

Keywords: public policies; childhood; mental health. 


\title{
PERFIL DE LOS NIÑOS ATENDIDOS EN EL AMBULATORIO DE SALUD MENTAL DE IÇARA - SC
}

\begin{abstract}
Resumen
El objetivo del siguiente estudio fue delinear el perfil de niños atendidos en el Ambulatorio de Salud Mental del municipio de Içara SC. Fue realizada una investigación documental de carácter exploratorio bajo el paradigma cuantitativo. Los datos relevados constaban en las historias clínicas de los niños de 6 a 11 años atendidos en el período de 2013 a junio de 2016. Los resultados muestran que el $58,3 \%$ eran del sexo masculino, el $38,3 \%$ tenían entre 8 y 9 años, el $27 \%$ eran derivados por sospechas de trastorno del neurodesarrollo. El $53 \%$ eran atendidos por la psicóloga, $31 \%$ recibian psicoterapia, el $21 \%$ usaban medicación y $12 \%$ psicoterapia asociada a los psicofármacos. Es necesario efectivar prácticas de perspectiva biopsicosocial, el conocimiento del perfil del usuario permite analizar las prácticas actuales y buscar intervenciones adecuadas.
\end{abstract}

Palabras claves: políticas públicas; infancia; salud mental.

\section{INTRODUÇÃO}

Foi a partir de 1988, com a promulgação da Carta Constitucional, marco da democracia e dos direitos da cidadania, que emerge no Brasil a necessidade de criar políticas e ações específicas para crianças e adolescentes, que até então, tinham os modelos de atenção dos adultos adaptados para eles (Sinibaldi, 2013). A Organização das Nações Unidas (ONU, 2012) estima que as crianças e adolescentes representem, respectivamente, cerca de $30 \%$ e $14,2 \%$ da população mundial, sendo que nesta faixa etária de desenvolvimento são encontradas altas taxas de prevalência de transtorno mental, em torno de 15,8\% (Thiengo, Cavalcante, \& Lovisi, 2014).

No Brasil, estudos epidemiológicos desenvolvidos em diversos estados e municípios, demostraram variações entre $7 \%$ e $24,6 \%$ de prevalência para algum transtorno mental em crianças e adolescentes (Garcia, 2015). Em um estudo recente sobre atendimentos infanto-juvenis em Centros de Atenção Psicossocial brasileiros, verificou-se que os principais diagnósticos são $21,4 \%$ para esquizofrenia, transtornos esquizotípicos e delirantes; 20,6\% para deficiência intelectual; $16,4 \%$ para transtornos do humor e 12,8\% para transtornos neuróticos, transtornos relacionados com o estresse e transtornos somatoformes (Garcia, 2015). Neste contexto, percebe-se a necessidade de serviços especializados para esta demanda, bem como de ampliação de processos de corresponsabilidade no cuidado e promoção da rede intersetorial.

Segundo Couto, Duarte e Delgado (2008) ainda se observa em vários países, desenvolvidos ou não, certa disparidade quanto à demanda de atenção em saúde mental infantil e a oferta de uma rede de serviço capaz de responder a ela. Esta discrepância se torna ainda mais evidenciada em países em desenvolvimento, como é o caso do Brasil. Neste sentido, os serviços ofertados em saúde mental, além de não satisfazerem as necessidades da demanda atendem nos moldes da população adulta, ou seja, não contemplam as 
especificidades da população infantil, pois é necessário estar atento às fases de desenvolvimento infantil e ao modo de intervenção (Couto et al, 2008).

De acordo com Couto et at. (2008), a população infanto-juvenil requer estratégias e serviços pensados propriamente a ela. Todavia, anteriormente aos anos 1980, o Brasil não dispunha de formulações e orientações do setor de saúde mental que guiassem a elaboração de uma rede de cuidados para a população infanto-juvenil com transtornos mentais ou sofrimento psíquico. Deste modo, essa função era outorgada a setores de assistência social e educação, que usavam medidas muito mais reparadoras e disciplinares do que de ordem clínica e psicossocial, se valendo, para tanto, de exclusão e violência (Couto et at., 2008; Taño \& Matsukura, 2015).

Couto e Delgado (2015) citam que foi por meio de serviços, como instituições do campo da assistência social e filantropia, educandários, reformatórios e outras instituições equivalentes, que se deu a institucionalização de crianças no Brasil, isto é, fora do sistema tradicional psiquiátrico. De acordo com Taño e Matsukura (2015) a assistência à infância e adolescência no Brasil foi excessivamente marcada por ações de proteção que resultaram em reclusão e institucionalização com evidentes situações de privação de direitos e liberdade.

De acordo com Taño e Matsukura (2015), apenas a partir da década de 1980 que se mudou totalmente a matriz de leitura para com a criança e os problemas dessa faixa etária. A criança passou a ser representada socialmente como um sujeito de direito, e passou a ter uma doutrina jurídica de proteção integral. O Estado deixou de ser um ente com função tutelar e de controle e passou a ter função de proteção e bem-estar social, e a proposta de intervenção deixou de ser a institucionalização em reformatórios e educandários e passou a ser o cuidado em liberdade, de orientação psicossocial.

Essa nova matriz de leitura sobre a criança e sobre as ações do Estado frente à mesma foi fruto de um período histórico marcado por muitas mudanças sociais, como: o movimento de redemocratização, a Constituição de 1988 e outras conquistas sociais dos anos 1980 e 1990 e, de forma particular, a Convenção Internacional sobre Direitos da Criança realizada pela ONU em 1989 e a promulgação do Estatuto da Criança e do Adolescente (ECA) (Couto \& Delgado, 2015).

Portanto, foi a partir das mudanças sociais ocorridas neste período no Brasil que se apresentou condições de possibilidades para a criação de um conjunto de orientações que formulam as atuais políticas públicas dirigidas às crianças e adolescentes, de acordo com os princípios éticos do Sistema Único de saúde (SUS), da Reforma Psiquiátrica e do ECA (Couto \& Delgado, 2015; Taño \& Matsukura, 2015). Desta forma, no início do século XXI houve o reconhecimento, pelas instâncias governamentais brasileiras, de que a saúde mental de crianças e jovens é questão de saúde pública e deve integrar o conjunto de ações do SUS (Couto et at., 2008). 
Seguindo esta perspectiva, a saúde mental de crianças e adolescentes foi tomando novos rumos e vislumbrando possibilidades de desenvolvimento como política pública de saúde mental alicerçada em bases éticas, políticas e clínicas a partir do ano de 2001. Este desenvolvimento se deu através da realização da III Conferência Nacional de Saúde Mental (CNSM) e a promulgação da Lei 10.216 (Presidência da República, 2001), que garante os direitos e a proteção das pessoas acometidas de transtorno mental e as assegura de seus direitos sem qualquer forma de discriminação quanto à raça, cor, sexo, orientação sexual, religião, opção política, nacionalidade, idade, família, recursos econômicos e ao grau de gravidade ou tempo de evolução de seu transtorno, ou qualquer outra (Couto \& Delgado, 2015).

Apenas dois meses depois da III CNSM, mais precisamente, em fevereiro de 2002, foi publicada a portaria 336/2002 (Ministério da Saúde, 2002). Nela continha um capítulo destinado somente à criação do Centro de Atenção Psicossocial para Crianças e Adolescentes (CAPSi), que se caracteriza como "um serviço de atenção diária destinado ao atendimento de crianças e adolescentes gravemente comprometidos psiquicamente" (Ministério da Saúde, 2004, p.23).

Neste sentido, os CAPSi constituem a primeira ação concreta advinda da nova posição do Estado brasileiro frente às questões de saúde mental infantil. Os CAPSi são compostos por equipes multiprofissionais e são alicerçados na lógica da atenção diária, na intensividade do cuidado, no trabalho em rede e de base territorial e comunitária. Sua função é estimular o exercício da cidadania para crianças e adolescentes que muitas vezes acabam afastadas de outros locais de sociabilidade em decorrência de um histórico de transtorno mental, ou seja, visam ampliar os laços sociais de seus usuários, familiares e comunidade. Para tanto, desenvolvem atividades como atendimento individual, atendimento grupal, atendimento familiar, visitas domiciliares, atividades de inserção social, oficinas terapêuticas, atividades socioculturais e esportivas, atividades externas. Sempre correspondente à faixa etária que se pretende atender (Couto \& Delgado, 2015; Taño \& Matsukura, 2015; Ministério da Saúde, 2004).

De acordo com Couto e Delgado (2015), os CAPSi devem exercer também a função de ordenadores da demanda de saúde mental infanto-juvenil no seu território de referência, contudo, salientam que os mesmos foram instituídos como dispositivos estratégicos na construção da rede de atenção psicossocial e não como serviços assistenciais especializados. Todavia, os CAPSi se constituem como referência para uma população de cerca de 200.000 habitantes, ou outro parâmetro populacional a ser definido pelo gestor local atendendo a critérios epidemiológicos (Ministério da Saúde, 2004). Nesta ótica, cidades com população menores devem buscar outras opções em serviços para atender a demanda infanto-juvenil de seu município.

Içara, município em que foi realizada a presente pesquisa não possui o número populacional adequado para construção do CAPSi, sendo assim utiliza o 
Ambulatório de Saúde Mental para oferecer atendimento à população infantojuvenil, dentro da rede de atenção à Saúde Mental da cidade. Essa rede de atenção é regulamentada pela portaria 3.088/2011 (Ministério da Saúde, 2011b) no modelo Rede de Atenção Psicossocial (RAPS) que se caracteriza por ser uma rede de atenção à saúde mental integrada e articulada nos diversos pontos de atenção para atender as pessoas em sofrimento, seja por transtornos mentais e/ou por uso drogas, sendo constituída pelos seguintes componentes: atenção básica em saúde, atenção psicossocial estratégica, atenção de urgência e emergência, atenção residencial de caráter transitório, atenção hospitalar, estratégias de desinstitucionalização, estratégias de reabilitação psicossocial (Ministério da Saúde, 2011b).

Entretanto, os Ambulatórios não constam como um dos serviços que compõe a RAPS (Ministério da Saúde, 2011b). Ainda assim muitos municípios os adotam como estratégia para suprir a falta de outros serviços. Os Ambulatórios são importantes pontos de atenção na rede de saúde mental e devem englobar os atendimentos realizados nas Unidades Básicas de saúde, centros de saúde e ambulatórios especializados. Tem como função desenvolver atividades centradas no atendimento individual ou em grupo, visitas domiciliares e atividades comunitárias, sobretudo na área de referência (Paiano, Maftum, Haddad, Marcon, 2016), e são regulamentados pela portaria/SNAS no 224 de 29 de janeiro de 1992 (Ministério da Saúde, 1992).

Sob a perspectiva da crescente demanda em saúde mental infantil, da dificuldade em apresentar redes de serviços especializadas a esta faixa etária e do pequeno número de pesquisas realizadas referentes a esta temática a proposta esta pesquisa. Deste modo, objetivou-se descrever o perfil das crianças atendidas no Ambulatório de Saúde Mental do município de Içara-SC, entendendo ser este o primeiro passo para repensar e efetivar novas estratégias de ações para ao público infantil.

\section{MÉTODO}

A pesquisa foi realizada no serviço de atendimento infantil do Ambulatório de Saúde Mental do município de Içara em Santa Catarina. O Ambulatório é vigente no município desde 2007, porém, o serviço de atendimento infantil somente se consolidou em 2013. O Ambulatório tem como objetivo prestar serviço de assistência à saúde mental para a população a nível ambulatorial e, para tanto, oferece o tratamento, a reabilitação, a reinserção social e a promoção da saúde mental. Sua clientela é composta de crianças a partir de 5 anos de idade até idosos, que possuam algum transtorno mental.

Esta pesquisa visou o atendimento infantil mais propriamente a crianças de 6 a 11 anos de idade, uma vez que se focou em uma única fase do desenvolvimento, no caso, a terceira infância (Papalia \& Feldman, 2013). Para o 
atendimento infantil, o Ambulatório conta com uma psicóloga e um médico psiquiatra, sendo os dois especializados em atendimentos a criança e adolescente.

A pesquisa se caracteriza como documental de abordagem quantitativa e de natureza exploratória (Gil, 2002; Marconi \& Lakatos, 2010; Michel, 2015), e tendo sido realizada partir dos prontuários dos usuários com idade entre 6 e 11 anos de idade atendidos no período de fevereiro de 2013 a junho de 2016, como detalhado: nas seções seguintes

\section{Participantes}

Este estudo coletou as informações de 180 prontuários de crianças com idade entre 6 a 11 anos, usuárias do Ambulatório de Saúde Mental do município de Içara no período de fevereiro de 2013 a junho de 2016.

\section{Instrumentos}

Tabela elaborada no programa Office Excel 2013 com as seguintes informações encontradas nos prontuários pesquisados: sexo, idade, motivo do encaminhamento, hipótese diagnóstica, profissionais consultados, tratamento e medicação.

\section{Procedimentos}

Inicialmente o projeto de pesquisa foi submetido e aprovado pelo Comitê de Ética em Pesquisa da Universidade do Extremo Sul Catarinense (CEP UNESC), número do parecer 1.785.773. Foram respeitados os termos das resoluções 196/96 revogada pela 466/2012-e 510/2016 do Conselho Nacional de Saúde.

Após autorização pela Instância da Gestão Municipal e pelo Ambulatório de Saúde Mental começou-se a transcrever as informações dos prontuários para uma tabela. Em seguida, os motivos de encaminhamento e as hipóteses diagnósticas de cada prontuário foram agrupados por transtorno mental, tendo como critério diagnóstico o Manual Diagnóstico e Estatístico de Transtornos (DSM-5) (APA, 2013) e a Classificação de Transtornos Mentais e de Comportamentos (CID-10) (OMS, 1993).

Análise dos dados

A análise dos dados foi de forma quantitativa. Para a caracterização da amostra, foi utilizada a estatística descritiva. Os dados coletados foram enumerados em uma tabela. Os mesmos foram quantificados, sendo o total de cada categoria (gênero, idade, motivo do encaminhamento, hipótese diagnóstica, profissionais consultados, tratamento e medicação) dividido pela sua respectiva quantidade. 


\section{RESULTADOS}

Entre os prontuários pesquisados no Ambulatório de Saúde Mental, constatou-se maior número de pacientes do sexo masculino, sendo que eles representaram $58,3 \%$ das crianças atendidas ( $41,7 \%$ foram do sexo feminino). Quanto à idade, 27,8\% tinham entre 6 a 7 anos de idade, 38,3\% entre 8 a 9 e $33,9 \%$ entre 10 a 11 anos.

A maior prevalência de encaminhamento de crianças ao Ambulatório foi por suspeita de Transtorno do Neurodesenvolvimento (30\%), em segundo lugar, o Transtorno Disruptivo, do Controle e da Conduta (20\%). Também houve encaminhamentos com suspeita de Transtorno de Ansiedade (16\%), Transtorno Depressivo (13\%) e para avaliação, sem nenhuma demarcação médica (14\%). Ainda, Transtornos Alimentares (4\%) e 3\% como signo de "outros" estão os Transtornos Obsessivo Compulsivo e associados (Transtorno do Neurodesenvolvimento com ansiedade e Transtorno do Neurodesenvolvimento com Transtorno Disruptivo do Controle e da Conduta). A maioria dos encaminhamentos derivam das Unidades Básicas de Saúde, feitas pelo clínico geral. A Figura 1 apresenta em gráfico os motivos de encaminhamento das crianças.

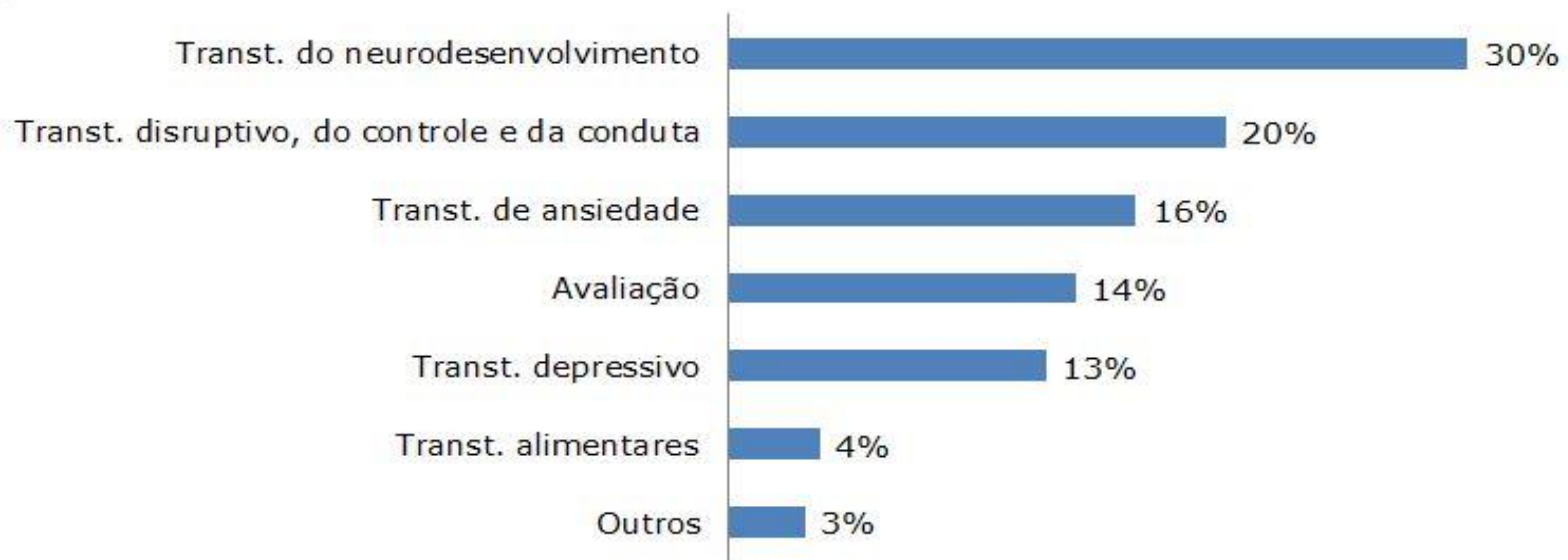

Figura 1. Motivos de encaminhamentos das crianças atendidas no Ambulatório de Saúde Mental do município de Içara - SC de 2013 a junho de 2016.

Também foram identificadas as hipóteses diagnósticas estabelecidas no Ambulatório pelos profissionais. Entretanto, 27\% dos prontuários pesquisados não continham esta informação, sendo assim, os resultados apresentados a seguir são referentes aos $73 \%$ de prontuários que continham informações sobre hipóteses diagnósticas. Deste modo, $42 \%$ das crianças encaminhadas para 0 Ambulatório não apresentaram nenhum quadro de transtorno, sendo os sintomas verificados pelas fontes de encaminhamento entendidos como um inadequado manejo da família para com a criança, por exemplo: falta de limites, questões de abuso de substâncias por algum familiar, separação dos pais, luto entre outros. Sendo assim, na maioria das vezes, o diagnóstico realizado pelo Ambulatório foi 
divergente com os encaminhamentos advindos em maior parte dos postos de saúde.

A segunda maior prevalência de hipótese diagnóstica foi de Transtorno do Neurodesenvolvimento (17\%), seguidos pelos: Transtorno de Ansiedade (15\%), Transtorno Disruptivo associado ao do Neurodesenvolvimento (12\%), Transtorno Disruptivo, do controle e da Conduta ( $8 \%$ ) e Transtorno depressivo e outros (TOC e Transtorno do Neurodesenvolvimento associado com ansiedade) com $3 \%$. A Figura 2 apresenta os dados em forma de gráfico.



Figura 2. Hipóteses diagnósticas das crianças atendidas no Ambulatório de Saúde mental do município de Içara - SC de 2013 a junho de 2016.

A equipe do serviço de atendimento infantil é composta por um psiquiatra e por uma psicóloga, ambos especializados em atendimento infantil. Quanto aos profissionais que fazem o atendimento a esses usuários, houve maior prevalência de atendimentos feitos pela psicóloga (53\%). Entretanto, esta conduz o processo de triagem e encaminhamentos, desta forma atendendo maior número de pacientes. Em seguida, $18 \%$ das crianças foram atendidas pela psicóloga e pelo psiquiatra concomitantemente e $15 \%$ apenas pelo psiquiatra. Além disso, $12 \%$ foram atendidas por outros profissionais que não fazem parte da equipe de atendimento infantil do Ambulatório, como neuropediatra e pediatra. Contudo, estes profissionais fazem parte da rede de saúde do município, e quando julgado necessário pelos profissionais do Ambulatório, as crianças são encaminhadas a eles.

No que se refere aos tratamentos ofertados pelo Ambulatório, 27\% dos prontuários não traziam esta informação. Com os restantes dos prontuários verificou-se que os tratamentos consistiam em psicoterapia (42\%), uso de psicofármacos $(28 \%)$, psicoterapia e psicofármaco juntos $(17 \%)$ e orientação familiar nos casos em que não há necessidade de tratamento (11\%).

Das crianças que utilizam psicofármacos, juntamente ou não com psicoterapia, identificou-se maior prescrição de metilfenidato associado com 
antipsicóticos (38\%), como mostra a Figura 3. Neste sentido observa-se que $52 \%$ das crianças que se utilizam de psicofármacos fazem uso do metilfenidato.

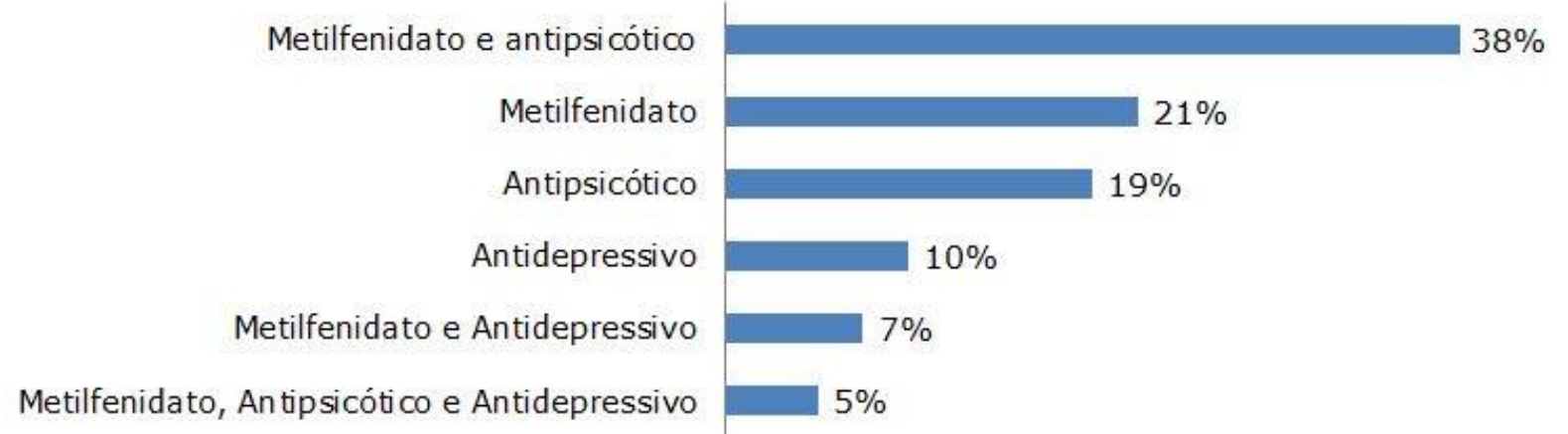

Figura 3. Prescrição de psicofármacos pelas crianças atendidas no Ambulatório de Saúde Mental do município de Içara - SC de 2013 a junho de 2016.

\section{DISCUSSÃO}

Este estudo teve por objetivo delinear o perfil das crianças atendidas no Ambulatório de Saúde Mental do município de Içara - SC, sendo que a pesquisa foi feita a partir dos prontuários das crianças de 6 a 11 anos de idade do período de fevereiro de 2013 a junho de 2016. Observou-se predominância do sexo masculino, de idade entre 8 e 9 anos e de encaminhamentos por Transtornos do Neurodesenvolvimento. Foi encontrada maior prevalência de atendimento feito pela psicóloga e tratamento psicoterápico, seguido pelo tratamento medicamentoso, sendo os mais prescritos o metilfenidato associado à antipsicóticos.

A maior prevalência encontrada foi do sexo masculino com $58,3 \%$, sendo que outros estudos também encontraram predominância deste, contudo em maiores proporções. Teixeira e Santos Jucá (2014) observaram em um CAPSi de Salvador que $72 \%$ dos pacientes eram do sexo masculino. Duarte, Santos, Schaefer, Lazzari e Lasta (2015) encontraram predominância de meninos $(70,17 \%)$ em um Ambulatório de Saúde Mental do Norte do RS, assim como no estudo de Goulart et al. (2016), (64,1\%) em um Ambulatório de Psiquiatria no Sul do Brasil. A faixa etária encontrada predominantemente foi de 8 a 9 anos, do mesmo modo que no estudo de Delvan, Portes, Cunha, Menezes e Legal (2010) no Serviço de Saúde Mental de Itajaí - SC. Entretanto, Garcia (2015) encontrou, em sua pesquisa nos CAPSi do território nacional, uma média de faixa etária com maior porcentagem na população entre 10 e 14 anos (41,3\%), seguido pela faixa de crianças entre 5 e 9 anos (30,9\%). Para o estudo de Garcia (2015), foram utilizados os dados dos Centros de Atenção Psicossocial com atendimentos de população infanto-juvenil disponibilizado pelo Departamento de informação do SUS, (APAC- DATASUS) entre 2008 e 2012. Foram obtidas informações sobre registros de atendimentos em relação a diagnósticos psiquiátricos, caraterísticas 
sociodemográficos do usuário e aspectos do atendimento oferecido. Para determinar o perfil nosológico do atendimento infanto-juvenil, foram analisados 144 CAPSi em todos os estados brasileiros.

A maior prevalência de encaminhamentos encontrada foi de Transtornos do Neurodesenvolvimento (30\%) que, segundo o DSM-5 (APA, 2013), se caracteriza por um grupo de sintomas que tem início na infância, normalmente antes de a criança ingressar na escola. Estes sintomas são evidenciados por prejuízos no funcionamento pessoal, social, acadêmico ou profissional. Abrange vários transtornos como, deficiências intelectuais, transtornos da comunicação (da linguagem, da fala, gagueira, etc.), transtorno do espectro autista, TDAH, transtorno específico da aprendizagem, transtorno motores e outros transtornos do neurodesenvolvimento. Já a CID-10 (OMS, 1993) denomina este grupo como Transtornos do Desenvolvimento Psicológico (F80-F89), que inclui transtornos específicos do desenvolvimento da fala e linguagem, das habilidades escolares, da função motora, transtornos específicos mistos do desenvolvimento, invasivo do desenvolvimento, outros transtornos do desenvolvimento e os não especificados.

Os Transtornos Disruptivo, de Controle e de Conduta foram observados em $20 \%$ dos prontuários, estes abarcam condições que envolvem problemas de autocontrole de emoções e comportamentos. Esses transtornos se iniciam na infância ou adolescência. Neste grupo, estão o transtorno de oposição desafiante, explosivo intermitente, da conduta e da personalidade antissocial, piromania e cleptomania além de outro transtorno disruptivo, do controle de impulsos e da conduta especificado e não especificado, segundo o DSM-5 (APA, 2013). A CID10 (OMS, 1993) traz especificamente dois grupos de transtornos desta classe, os transtornos de conduta (F91) e os transtornos misto de conduta e emoção (F92).

Contudo, as hipóteses diagnósticas estabelecidas pelos profissionais do local foram, em sua maioria de ausência de qualquer transtorno (42\%), sendo atribuídos os sintomas a um manejo familiar inadequado. O DSM-5 (APA, 2013) classifica esta questão como "condições que podem ser foco da atenção clínica", uma vez que não são transtornos mentais, porém podem agravar um quadro de transtorno ou qualquer problema médico, assim como afetar no atendimento, tratamento e prognóstico do paciente. Entram neste grupo os problemas de relacionamento entre pais e filhos ou com irmãos, a distância dos pais, brigas ou separação destes. Inclui-se também neste grupo o luto e abuso e negligência com a criança estando dentro os maus tratos, violência, abuso sexual ou psicológico. A CID-10 (Organização Mundial de Saúde, 1993) também classifica estes problemas denominando-os como "fatores influenciando o estado de saúde e contato com serviço de saúde", que tratam especificamente de problemas ligados ao meio familiar. Estes fatores são colocados sob os códigos Z61, Z62 e Z63, sendo classificados, respectivamente, como problemas relacionados a eventos de vida negativos na infância, outros problemas relacionados à criação e 
outros problemas relacionados ao grupo de suporte primário, incluindo circunstâncias familiares.

Papalia e Feldman (2013) citam a pesquisa que Kaczynski desenvolveu com 226 famílias com filhos em idade escolar demostrando que crianças expostas à discórdia parental e/ou parentalidade insatisfatória tendiam a desenvolver altos níveis tanto de comportamentos internalizantes, como medo e humor deprimido, quanto de comportamento externalizante, como agressividade, brigas, hostilidade, entre outros. No entanto, é importante salientar que estes problemas de comportamento também devem receber tratamento, uma vez que os ambientes social e psicológico influenciam fortemente na saúde mental da criança mais do que as próprias características intrínsecas do sujeito (Halpern \& Figueiras, 2004).

Ainda sobre a hipótese diagnóstica, D'Abreu (2012) refere-se à preocupação de muitos profissionais de fazerem um diagnóstico da criança tendo por base manual demasiado categórico, estático e restritivo, não levando em conta as singularidades de cada pessoa, sendo pouco sensível às especificidades do desenvolvimento característico da infância. Outra preocupação que se faz presente é a consequência do "rótulo psiquiátrico", que por vezes ajuda na manutenção do transtorno e da visão do problema centrada no indivíduo em detrimento do coletivo.

Na pesquisa de Gonzalez (2016) verificou-se que o estigma psiquiátrico vai aumentando conforme a idade da criança, assim como sua nocividade. Além disso, o estigma pode fazer com que o transtorno evolua drasticamente, bem como gerar outros transtornos psiquiátricos, podendo privar o indivíduo de uma relação saudável consigo mesmo (baixa autoestima) e outras relações sociais importantes para o desenvolvimento. O estigma também pode construir barreiras no processo de procura e participação de tratamentos em saúde mental e na distribuição de oportunidades de vida de modo geral.

A segunda maior prevalência, quanto à hipótese diagnóstica do local, foi o Transtorno do Neurodesenvolvimento ou F80-F89 (OMS, 1993) com 17\%. Dentre os Transtornos do Neurodesenvolvimento o TDAH vem se destacado fortemente. Gonçalves, Pureza e Prando (2011) explanam que a frequência dessa patologia é cada vez mais alta no Brasil, sendo que em crianças em idade escolar a prevalência varia de $5 \%$ a $18 \%$. O TDAH é um dos transtornos mais diagnosticados na atualidade, com alta predominância na população infantil e, além disso, os sintomas compatíveis a ele se mostram como uma das principais causas de procura por atendimento em serviços de saúde mental para crianças e adolescentes.

Do mesmo modo, em uma pesquisa nacional em CAPSi com crianças e adolescentes de 5 a 19 anos, Garcia (2015) constatou que 23,6\% possui diagnóstico de Transtornos do Desenvolvimento Psicológico (F80-F89), e 12,5\% de Retardo Mental (F70 - F79), tais diagnósticos, quando entendidos pela ótica 
do DSM-5 (APA, 2013) fazem parte do grupo de Transtornos do Neurodesenvolvimento.

Já o transtorno de ansiedade, que se caracteriza como um problema de comportamento internalizante e que tende a ser maior em crianças escolares (Bolsoni-Silva, Loureiro, \& Marturano, 2016), também atinge parte das crianças usúarias do Ambulatório (15\%), e alcançou a segunda maior incidência na pesquisa de Delvan et al. (2010) com crianças que utilizam o serviço de saúde mental em Itajaí - SC. Este grupo inclui os transtornos de ansiedade de separação, mutismo seletivo, fobia social, fobia específica, transtorno de pânico, agorafobia, transtorno de ansiedade generalizada, transtorno de ansiedade devido a uma condição médica geral, transtorno de ansiedade induzido por substância e transtorno de ansiedade especificado e sem outra especificação (APA,2013), também podem ser denominados transtornos fóbicos-ansiosos (F40) e outros transtornos ansiosos (F41) (OMS, 1993).

Quanto aos profissionais consultados, verificou-se predominância de atendimentos psicológicos (53\%) em relação aos psiquiátricos (15\%), diferentemente da pesquisa de Goulart et al. (2016) que encontrou a maioria dos atendimentos prestados feitos por médico psiquiatra (76,3\%). Foi identificado também que $18 \%$ das crianças recebem atendimento psicológico e psiquiátrico concomitantemente, combinando diferentes tipos de tratamentos.

Caminha, Wainer, Oliveira e Piccoloto (2003) ressaltam que a psicoterapia combinada com psicofármacos tem se mostrado muito eficaz no tratamento de diversos transtornos mentais, uma vez que em determinadas situações, mesmo com diagnósticos realizados em psicoterapia, precisa-se de uso de medicações psiquiátricas para diminuição dos sintomas emergentes. Sendo assim, quando administrado adequadamente, ou seja, com o acompanhamento adequado, essa associação pode colaborar eficientemente para melhora da qualidade de vida do paciente assim como para a evolução do seu quadro psiquiátrico.

Por fim, quanto à medicação, a maior prescrição foi de metilfenidato, o psicoestimulante mais consumido atualmente no mundo, mais do que a soma de todos os outros estimulantes existentes. No Brasil não é diferente, sendo que o metilfenidato é o estimulante mais consumido e este consumo vem crescendo a cada ano. Depois dos Estados Unidos, o Brasil é o segundo país que mais consome Ritalina (Decotelli, Teixeira, Paulo \& Bicalho, 2013; Ortega et al., 2010).

Em contrapartida, Decotelli et al. (2013) criticam a utilização exacerbada deste medicamento e afirmam que a prescrição deveria ser exceção e não indiscriminadamente como acontece atualmente. Os autores defendem que milhares de crianças são medicadas para ter o máximo de aproveitamento de suas capacidades cognitivas. Além disso, Caminha et al. (2003) chamam a atenção para a "medicalização" do sofrimento humano e fazem ressalvas quanto 
a diagnósticos precoces e medicações inadequadas, que acabam por prejudicar o paciente mais do que Ihe garantir algum benefício, mesmo que a longo prazo.

Entende-se que a criança tem necessidades muito singulares, exclusivas da sua condição de infante, sendo assim, a oferta de serviços específicos para esse público mostra-se cada vez mais necessária, indo ao encontro do aumento da demanda infantil na rede de saúde mental. Porém, há uma lacuna entre a oferta de serviço em saúde mental para essa população e a demanda carente por ela, ou seja, os serviços ofertados não dão conta da demanda que precisa ser atendida, uma vez que estes são poucos em relação à população que carece deste atendimento. Além disso, sabe-se que são recentes as discussões sobre o atendimento psiquiátrico e psicológico em saúde mental infantil na rede pública e estratégias terapêuticas direcionadas a este público, assim é necessário caminhar em direção à melhores delineamentos das práticas voltadas a essa população já que a necessidade dela se torna, gradativamente, maior com o passar do tempo (Couto \& Delgado, 2015).

No Ambulatório pesquisado, esta lacuna, entre demanda e oferta de serviço em saúde mental infantil, pode ser observada. A instituição conta com uma fila de espera para o serviço de atendimento infantil e poucos profissionais especializados para atendê-la, ou seja, a necessidade é maior do que os recursos humanos disponíveis para atendimento. Todavia, cabe informar que ao término da coleta de dados outro psicólogo havia sido contratado para o Serviço de Atendimento Infantil.

Através do delineamento do perfil do usuário, é possível iniciar estratégias de promoção, prevenção e produção de saúde para o município em questão. Estas estratégias teriam a finalidade de trabalhar para a solução dos problemas e não apenas ao tratamento dos sintomas, ou seja, buscando possibilidades de ações com ênfase na prevenção primária. Além disso, pode-se pensar em uma assistência a partir do paradigma biopsicossocial, que entende a saúde como sendo construída nas relações com o outro e consigo mesmo (Pereira, Barros \& Augusto, 2011), buscando promover ao usuário aquilo que já lhe é de direito e práticas que possam diminuir o sofrimento deste, dentro de uma lógica que visa à necessidade de reinventar novas práticas de cuidado.

\section{CONSIDERAÇÕES FINAIS}

Muitas conquistas marcaram o campo da saúde mental infantil no Brasil e no mundo, contudo ainda existem muitos progressos que precisam ser feitos. Com esta ótica, este estudo objetivou delinear o perfil das crianças usuárias do Ambulatório de Saúde Mental do município de Içara, compreendendo a criança como sujeito de direito e de especificidades próprias inerente à sua condição e fase do desenvolvimento. 
Uma limitação observada na coleta de dados nesta pesquisa foi a falta de informação nos prontuários. Sendo assim, este estudo também suscitou a importância do correto preenchimento dos prontuários, uma vez que o registro das informações em instituições públicas de saúde torna viável a condução de pesquisas de caráter epidemiológico, permitindo uma melhor análise da mesma, assim como avaliações e planejamentos relativos à gestão municipal. Ademais, todo usuário e os familiares deste têm o direito de ter acesso às informações contidas em seu prontuário (Ministério da Saúde, 2011a), sendo assim, estas informações devem estar corretamente registradas.

\section{DECLARAÇÃO DE CONFLITOS DE INTERESSE}

Não há conflitos de interesse.

\section{REFERÊNCIAS}

Associação Psiquiátrica Americana. (2013). Transtorno do Neurodesenvolvimento. Manual Diagnóstico e Estatístico de Transtornos Mentais ( $5^{\mathrm{a}}$ ed.). Arlington, VA: American Psychiatric Publishing.

Bolsoni-Silva, A. T., Loureiro, S. R., \& Marturano, E. M. (2016). Comportamentos internalizantes: Associações com habilidades sociais, práticas educativas, recursos do ambiente familiar e depressão materna. Psico, 47(2), 111-120. doi: $10.15448 / 1980-8623.2016 .2 .20806$.

Caminha, R. M., Wainer, R., Oliveira, M., \& Piccoloto, N. (2003). Psicoterapias cognitivo-comportamentais: Teoria e prática. São Paulo, SP: Casa do Psicólogo.

Couto, M. C. V., \& Delgado, P. G. G. (2015). Crianças e adolescentes na agenda política da saúde mental brasileira: Inclusão tardia, desafios atuais. Psicologia Clínica, 27(1), 17-40. doi:10.1590/0103-56652015000100002

Couto, M. C. V., Duarte, C. S., \& Delgado, P. G. G. (2008). A saúde mental infantil na Saúde Pública brasileira: Situação atual e desafios. Revista Brasileira de Psiquiatria, 30(4), 390-398. doi:10.1590/S151644462008000400015.

D'Abreu, L. C. F. (2012). O desafio do diagnóstico psiquiátrico na criança. Contextos Clínicos, 5(1), 2-9. doi:10.4013/ctc.2012.51.01

Decotelli, K. M., Bohre, L. C. T., \& BICALHO, P. P. G. (2013). A droga da obediência: Medicalização, infância e biopoder - notas sobre clínica e política. Psicologia Ciência e Profissão, Brasília, 33(2), 446-459. doi: $10.1590 /$ S1414-98932013000200014. 
Delvan, J. D. S., Portes, J. R. M., Cunha, M. P., Menezes, M., \& Legal, E. J. (2010). Crianças que utilizam os serviços de saúde mental: Caracterização da população em uma cidade do sul do Brasil. Revista Brasileira de Crescimento e Desenvolvimento Humano, 20(2), 228-237. Recuperado em 30 de abril de 2019, de

http://pepsic.bvsalud.org/scielo.php?script=sci arttext\&pid=S0104-

$12822010000200006 \&$ lng $=p t \& t \operatorname{lng}=p t$.

Duarte, C. P., Santos, A. S. A., Madeira, K. H., Lazzari, A., \& Lasta, J. B. (2015). Perfil epidemiológico das crianças atendidas no ambulatório de saúde mental de uma cidade do litoral norte do RS. Revista de Iniciação Científica da ULBRA, 1(13), 71-79. Recuperado em 30 de abril de 2019, de http://www.periodicos.ulbra.br/index.php/ic/article/view/1449/1194.

Garcia, G. Y. C. (2015). Panorama de assistência em saúde mental infantojuvenil em Centros de Atenção Psicossocial no Brasil. (Dissertação de Mestrado). Universidade Federal da Bahia, Salvador. Recuperado em 30 de abril de 2019, de https://repositorio.ufba.br/ri/bitstream/ri/17913/1/Diss\%20Final.\%20Grey \%20Yuliet\%20Garcia.\%20\%202015.pdf.

Gil, A. C. (2002). Como elaborar projetos de pesquisa. São Paulo, SP: Atlas.

Gonçalves, H. A., Pureza, J. R., \& Prando, M. L. (2011). Transtorno de déficit de atenção e hiperatividade: Breve revisão teórica no contexto da neuropsicologia infantil. Neuropsicologia Latinoamericana, 3(3), 20-24. doi: $10.5579 / \mathrm{rnl} .2011 .0076$.

Gonzalez, A. F. C. (2016). O estigma das perturbações mentais: Um estudo qualitativo pela voz das crianças. (Dissertação de Mestrado). Instituto Universitário Ciências Psicológicas, sociais e da vida. Recuperado de http://repositorio.ispa.pt/bitstream/10400.12/5066/1/9439.pdf.

Goulart, A. P., Jordani, V., Sakae, T. M., Sakae, G. R. F. M., Schaefer, T. F., \& Meyer, H. F. (2016). Perfil epidemiológico de crianças e adolescentes atendidos em um ambulatório de psiquiatria no sul do Brasil no período de 2004 a 2012. Arquivos Catarinenses de Medicina, 45(3), 17-34. Recuperado em 20 de abril de 2019, de http://www.acm.org.br/acm/seer/index.php/arquivos/article/view/108/99.

Hales, R. \& Yudofsky, S. (2004). Tratado de Psiquiatria Clinica. Espanha, Barcelona: Masson.

Halpern, R., \& Figueiras, A. C. (2004). Influências ambientais na saúde mental da criança. Jornal de Pediatria, 80(2), 104-110. doi:10.1590/S002175572004000300013. 
Ministério da Saúde. Agência Nacional de Vigilância Sanitária (2011a). Resolução de Diretoria Colegiada - Rdc no 63, de 25 de novembro de 2011. Dispõe sobre os requisitos de boas práticas de funcionamento para os Serviços de

Saúde. Recuperado em 30 de abril de 2019, de http://portal.anvisa.gov.br/documents/33880/2568070/rdc0063 251120 11.pdf/94c25b42-4a66-4162-ae9b-bf2b71337664.

Ministério da Saúde. Gabinete do Ministro (2002). Portaria no 336, de 19 de fevereiro de 2002. Recuperado em 30 de abril de 2019, de http://bvsms.saude.gov.br/bvs/saudelegis/gm/2002/prt0336 19022002. html.

Ministério da Saúde. Gabinete do Ministro (2011b). Portaria no 3.088, de 23 de dezembro. Institui a Rede de Atenção Psicossocial para pessoas com sofrimento ou transtorno mental e com necessidades decorrentes do uso de crack, álcool e outras drogas, no âmbito do Sistema Único de Saúde (SUS). Brasília-DF: Diário Oficial da União, n. 96.

Ministério da Saúde. Secretaria de Atenção à Saúde. Departamento de Ações Programáticas Estratégicas. (2004). Saúde Mental no SUS: Os centros de atenção psicossocial. Brasília, DF: Autor. Recuperado em 30 de abril de 2019, de http://www.ccs.saude.gov.br/saude mental/pdf/sm sus.pdf.

Ministério da Saúde. Secretaria Nacional de Assistência à Saúde (1992). Portaria/SNAS no 224, de 29 de janeiro de 1992. Estabelece diretrizes e normas para o atendimento em saúde mental. Brasília-DF: Diário Oficial da União 1994, n. 147.

Michel, M. H. (2005). Metodologia e pesquisa científica em ciências sociais: Um guia para acompanhamento da disciplina e elaboração de trabalhos monográficos. São Paulo, SP: Atlas.

Marconi, M. D. A., \& Lakatos, E. M. (2010). Fundamentos de metodologia científica. São Paulo, SP: Atlas.

Organização das Nações Unidas. (2012). Objetivos de desenvolvimento do milênio: Mapa do progresso de 2012. Nova York, NY: Divisão de Estatística do Departamento de Assuntos Econômicos e Sociais.

Organização Mundial da Saúde. (1993). Classificação de transtornos mentais e de comportamento da CID-10: Descrições e diretrizes diagnósticas. Porto Alegre, RS: Artes Médicas.

Ortega, F, Barros, D., Caliman, L., Itaborahy, C., Junqueira, L., \& Ferreira, C. P. (2010). A Ritalina no Brasil: Produções, discursos e prática. Interface, 14(34), 499-510. doi:10.1590/S1414-32832010005000003.

Paiano, M., Maftum, M., Haddad, M. C. L., Marcon, S.S. (2016) Ambulatório de Saúde Mental: Fragilidades apontadas por profissionais. Texto \& ContextoEnfermagem, 25(3). doi: 10.1590/0104-07072016000040014.

Papalia, D. E., \& Feldman, R. D. (2013). Desenvolvimento humano. (12a ed.). Porto Alegre, RS: Artmed. 
Pereira, T. T. S. O., Barros, M. N. D. S., \& Augusto, M. C. N. D. A. (2011). O cuidado em saúde: O paradigma biopsicossocial e a subjetividade em foco. Mental, 9(17), 523-536. Recuperado em 30 de abril de 2019, de http://pepsic.bvsalud.org/scielo.php?script=sci arttext\&pid=S1679$44272011000200002 \& \operatorname{lng}=p t \& \operatorname{lng}=p t$.

Presidência da República. Casa Civil. Subchefia para Assuntos Jurídicos (2001). Lei n. 10.216, de 6 de abril de 2001. Dispõe sobre a proteção e os direitos das pessoas portadoras de transtornos mentais e redireciona o modelo assistencial em saúde mental. Recuperado em 30 de abril de 2019, de http://www.planalto.gov.br/ccivil 03/leis/leis 2001/l10216.htm.

Sinibaldi, B. (2013). Saúde mental infantil e atenção primária: Relações possíveis. Revista de Psicologia da UNIFESP, 12 (2), 60-72.

Taño, B. L., \& Matsukura, T. S. (2015). Saúde mental infanto-juvenil e desafios do campo: Reflexões a partir do percurso histórico. Cadernos de Terapia Ocupacional da UFSCar, 23(2), 439-447. doi:10.4322\%2F01044931. ctoAR0479

Teixeira, R. M., \& dos Santos Jucá, V. J. (2014). Caracterização dos usuários de um centro de atenção psicossocial infanto-juvenil do município de Salvador (BA). Revista de Psicologia, 5(2), 70-84. Recuperado em 30 de abril de 2019, de http://www.periodicos.ufc.br/psicologiaufc/article/view/1477/1375.

Thiengo, D.L., Cavalcante, M.T., \& Lovisi, G.M. (2014). Prevalência de transtornos mentais em crianças e adolescentes fatores associados: uma revisão sistemática. Jornal Brasileira de Psiquiatria. 63(4), 360-372. doi.org/10.1590/0047-2085000000046

\section{Sobre os autores}

Maísa Pedro da Silva Serafim é psicóloga pela Universidade do Extremo Sul de Santa Catarina. maisa@unesc.net

Karin Martins Gomes é psicóloga pela Universidade do Vale do Itajaí, mestre e doutora em Ciências da Saúde pela Universidade do Extremo Sul Catarinense, docente e coordenadora do curso de Psicologia da Universidade do Extremo Sul Catarinense. karin@unesc.net

Dipaulla Minotto da Silva é psicóloga pela Universidade do Extremo Sul de Santa Catarina, mestre em Saúde Coletiva pela Universidade do Extremo Sul de Santa Catarina e docente do curso de Psicologia da Universidade do Extremo Sul de Santa Catarina.dms@unesc.net

João Luiz Brunel é psicólogo pela Universidade Federal de Santa Catarina, mestre em Educação pela Universidade do Sul de Santa Catarina e docente do curso de Psicologia da Universidade do Extremo Sul de Santa Catarina. jlb@unesc.net 
Certificamos que todos os autores participaram suficientemente do trabalho para tornar pública sua responsabilidade pelo conteúdo. A contribuição de cada autor pode ser atribuída como se segue: Maísa Pedro da Silva Serafim e Karin Martins Gomes, contribuíram para a conceitualização, investigação e visualização do artigo, João Luiz Brunel e Dipaula Minotto da Silva fizeram a redação inicial do artigo (rascunho) e Maísa Pedro da Silva, Karin Martins Gomes, João Luiz Brunel e Dipaula Minotto da Silva . são os responsáveis pela redação final (revisão e edição).

Os autores agradecem à Prefeitura Municipal do Município de Içara SC, à Secretaria de Saúde do Município de Içara e ao Ambulatório de Saúde Mental do Município de Içara pelo apoio na coleta de dados.

Recebido em: 11/07/2017

$1^{\text {a }}$ revisão em: 17/09/2017

Aceito em: 08/03/2018 